\title{
Towards a High Accuracy Wearable Hand Gesture Recognition System Using EIT
}

\author{
Yu Wu${ }^{1}$, Dai Jiang ${ }^{1}$, Jifang Duan ${ }^{1}$, Xiao Liu ${ }^{1}$, Richard Bayford ${ }^{2}$, and Andreas Demosthenous ${ }^{1}$ \\ ${ }^{1}$ Department of Electronic and Electrical Engineering, University College London, Torrington Place, London WC1E 7JE, UK \\ ${ }^{2}$ Department of Natural Sciences, Middlesex University, The Burroughs, London, NW4 4BT, UK \\ e-mail: yu.wu.09@ucl.ac.uk; a.demosthenous@ucl.ac.uk
}

\begin{abstract}
This paper presents a high accuracy hand gesture recognition system based on electrical impedance tomography (EIT). The system interfaces the forearm using a wrist wrap with embedded electrodes. It measures the inner conductivity distributions caused by bone and muscle movement of the forearm in real-time and passes the data to a deep learning neural network for gesture recognition. The system has an EIT bandwidth of 500 $\mathrm{kHz}$ and a measured sensitivity in excess of $6.4 \Omega$ per frame. Nineteen hand gestures are designed for recognition, and with the proposed round robin sub-grouping method, an accuracy of over $98 \%$ is achieved.
\end{abstract}

\section{INTRODUCTION}

Hand gesture recognition is an attractive communication method that can be widely used for human-computer interaction (HCI). This seamless link between humans and machines can enhance quality of life, with applications ranging from better controlling a smart device, safely handling hazardous material to restoring a degree of normality to amputees. Given the exciting potential of the HCI link, many systems have been reported [1]. They comprise two building blocks: the user intention capturing (UIC) block, which is the main focus of this work, and the feature extraction and classification or machinelearning block. Machine operation can be implemented after a classification is given.

One popular UIC method is camera-assisted hand gesture recognition [2]; a picture is taken for a single gesture and passed on for classification. This UIC method is simple but with many practical limitations, such as requirement for line-of-sight, and application constraints e.g. not suitable for building a prosthetic hand. An alternative UIC method is electromyography (EMG), as most of the muscles responsible for hand motion are within the forearm. With skin preparation and careful electrode placement, voltage potentials ranging up to tens of $\mathrm{mV}$ with frequencies up to $500 \mathrm{~Hz}$ can be measured near these muscles when they contract. Because different motions activate different muscle groups, successive recorded data can be used for gesture classification [3], [4].

Electrical impedance tomography (EIT) is a non-invasive bio-impedance measurement method that can be used to estimate the inner structural conductivity distribution of any enclosed conductive object. EIT has been developed mainly as an imaging technique for biomedical applications such as cancer detection and lung aeration monitoring. Typically, an array of electrodes in the shape of a band is wrapped around the subject under test (SUT), and by injecting ac currents through a

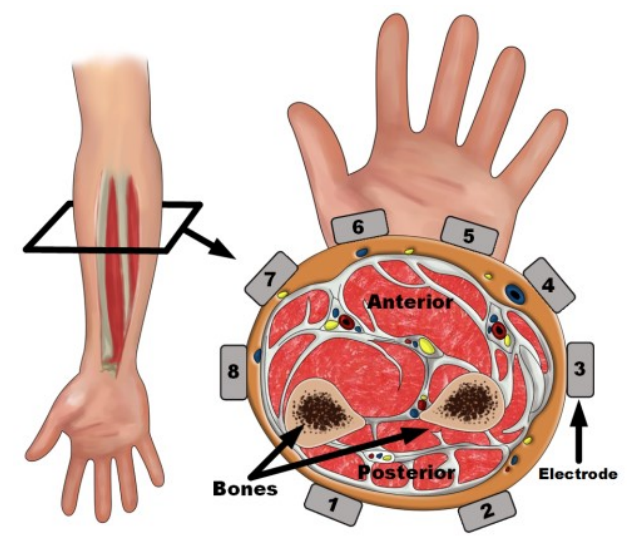

Fig. 1. Cross-section of the forearm with position of electrodes.

pair of electrodes, voltage potentials are developed and can be measured differentially around the SUT. Using a defined injection and measurement sequence, a set of data can be collected for conductivity distribution estimation. While a hand gesture is performed, bones and muscles move under the skin resulting in a temporal alteration of conductivity distribution which can be measured by EIT for recognition. Compared to EMG, EIT may offer more design flexibility such as signal amplitude and frequency, which is related to the excitation source, number of electrodes in the array, and measurement pattern.

A wearable EIT system for hand gesture recognition is reported in [5], [6]. These studies show good results, but the hardware performance (e.g. EIT bandwidth) is limited by the AD5933, a commercial bio-impedance analyzer integrated circuit (IC) using two-electrode measurements. Although the hardware in [6] implemented a four-electrode measurement scheme, it employs a single-ended current driver that produces large common-mode voltages on the measuring side [7]. Moreover, the system does not produce impedance values with real and imaginary parts, which is often more useful than the measured voltage RMS value.

This paper presents a high performance EIT system using commercial ICs, complemented by a deep auto-encoder neural network for implementing hand gesture recognition. It features a novel common-mode feedback (CMFB) current driver driven by a high-speed direct digital synthesizer (DDS) signal generator, and high-resolution digital I-Q demodulators. The rest of the paper is organized as follows. Section II presents the system design architecture and Section III the system 


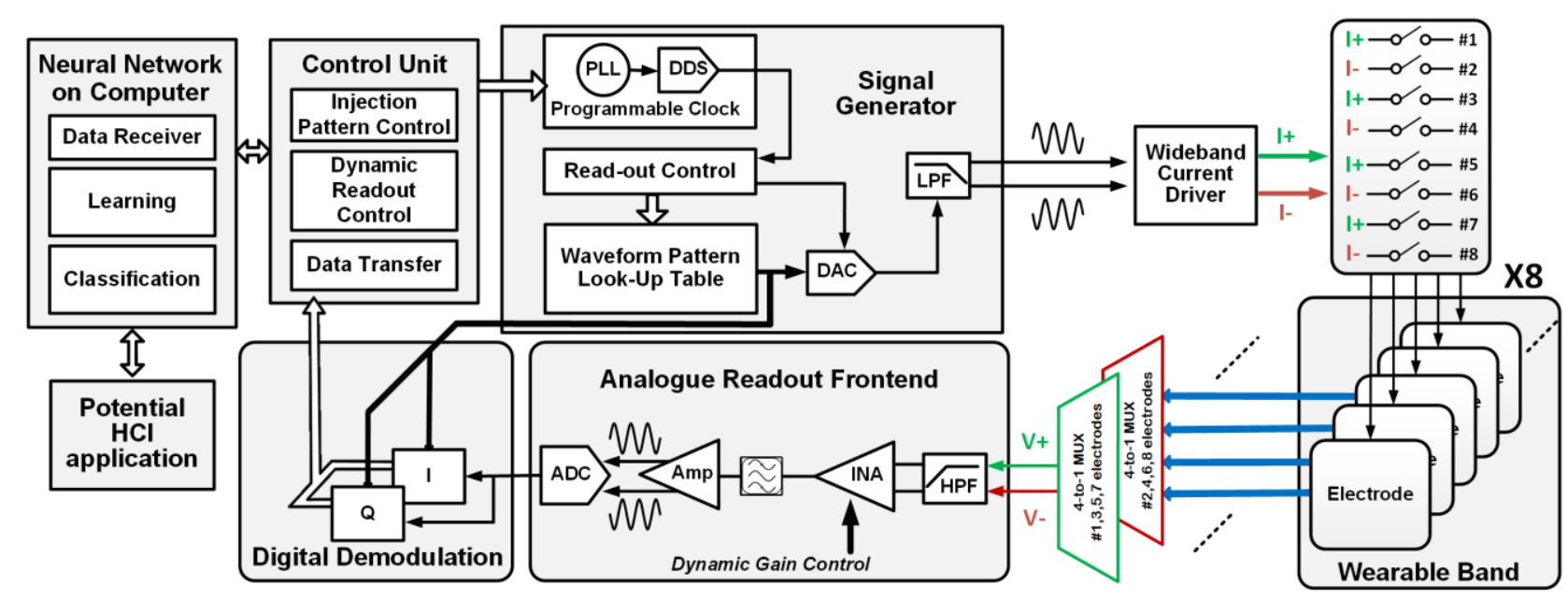

Fig. 2. Top-level system architecture.

implementation with circuit details. Section IV presents measured results and Section V concludes the paper.

\section{SYSTEM ARCHITECTURE}

Hand motion is heavily related to the physical movement of muscles and bones in the forearm. The proposed system aims to measure these movements using EIT technology. The system measures and represents these movements in terms of bioimpedance distribution alterations in the cross-section plane that the electrode-band is wrapped around. A cross-section view of the forearm is shown in Fig. 1. The forearm muscles that control hand motions can be generally grouped into flexors in the anterior surface, and extensors in the posterior surface surrounding the radius and ulna bones. As the system detects a slice of the forearm, band position and electrode array distribution play a critical role in high accuracy recognition. Mid-forearm measured half hand distance away from the elbow and the wrist are the preferred band positions. The former is where the belly of these muscles lie, and towards muscle tendons change can be observed for the latter. As wrist position may be unavailable for some applications, mid-forearm is chosen as the band position for this study. Eight electrodes are chosen to ensure easy and firm electrode contact and are placed as shown in Fig. 1 to high EIT mesh to the flexors.

Fig. 2 shows the overall high-level system architecture. Based on the principle of EIT, the system can be divided into the current excitation and voltage measurement parts. A voltage signal is generated with a digital-to-analog converter (DAC) driven by a look-up table (LUT) based DDS. This signal is fed to a voltage controlled current source (current driver). The current driver is required to be floating so that the load is driven differentially. This reduces the common-mode signal and gives optimum sensitivity for the voltage amplifier to detect small impedance changes. The voltage measurement consists of an ac coupled instrumentation amplifier with dynamic gain controls, followed by a band-pass filter and a gain amplifier that interfaces to an analog-to-digital converter (ADC). After the FPGA receives a trigger signal from the $\mathrm{PC}$, it initiates the $\mathrm{DAC}$ and controls the analog switches according to adjacent EIT drive pattern [6], then takes the $\mathrm{ADC}$ results for $\mathrm{I}-\mathrm{Q}$ demodulation.

For adjacent EIT drive pattern with four-electrode measurements, $4 \times N$ number of analog switches are often used, where $N$ is the total number of electrodes. In this design, the odd or even numbered of electrodes are connected by 4 SPST switches to a single node; this enables the adjacent EIT drive method but halves the number of switches required. Not only does this reduce the parasitic capacitance in parallel with the output node of the current driver, but it also enables a symmetrical layout at the input of the instrumentation amplifier, so a higher CMRR can be achieved. Because of this switch interconnection, certain voltage measurements are 180 degrees out of phase to the waveform in the look-up table, which can be easily inverted in the digital domain before demodulation. Demodulated data is transferred to a PC for processing of hand gesture recognition.

\section{SYSTEM IMPLEMENTATION}

\section{A. Current Excitation}

The detailed circuit schematic is shown in Fig. 3. The FPGA SPI communicates with a 16-bit, high-speed DAC to generate a voltage signal drive excitation. The coarse sinewave is applied to amplifier low-pass filter the output of which excites the current driver. The default excitation frequency is $200 \mathrm{kHz}$ with a filter cut-off frequency around $350 \mathrm{kHz}$, but the frequency can vary up to $500 \mathrm{kHz}$ if required.

In a high performance EIT system, the current driver is required to be fully differential to reduce the common mode signal. However, any mismatch or imbalance between the current source and sink parts also generates a common-mode signal that adds to the small signals measured by the instrumentation amplifier [7]. Many studies have reported novel current drivers for EIT using CMOS technology [8], [9], however the mismatch-sensitive Howland current driver is still commonly adapted in discrete EIT systems. In this work, a master-slave current driver is presented that employs a CMFB 


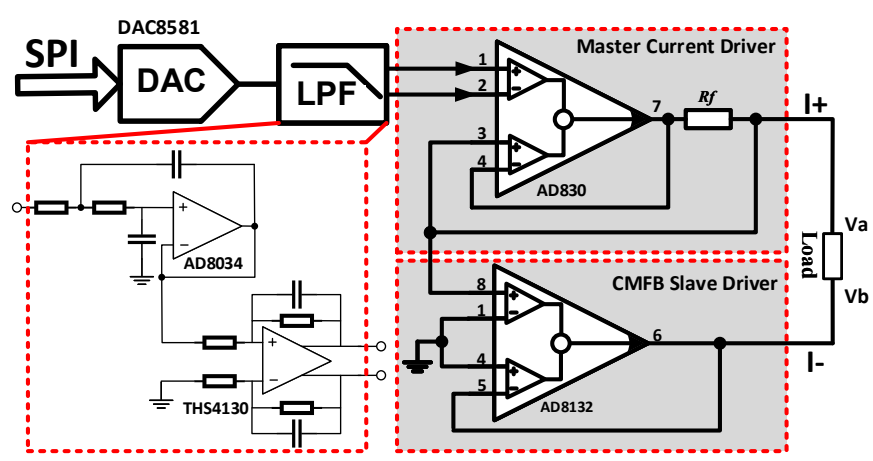

Fig. 3. Detailed current injection circuit schematic of the EIT system

technique to minimize the mismatches and thus reduce common-mode errors.

The sourcing current is provided by an AD830 configured in a resistive feedback topology. Its transfer function can be written as:

$$
G m=\frac{\text { Iout }}{\text { Vin }}=\frac{A_{\text {open-loop }}}{R_{f}+R_{\text {load }}+A_{\text {open-loop }} R_{f}} \approx \frac{1}{R_{f}} .
$$

The master outputs a constant current that is independent of the load. The CMFB slave driver AD8132 measures the voltages across the load through pins 8 and 5, and by connecting pins 1 and 4 to ground to form a feedback loop. This feedback loop adjusts the output voltage $\mathrm{V}_{\text {pin-6 }}$ until the common-mode voltage across the load is zero. Consequently, the slave produces a voltage that is differential to the output of the AD830; thus it sinks the same amount of current that is sourced by the master. This feedback can be also seen as a resistor-less inverter with its transfer function written as:

$$
V_{\text {pin-8 }}=\mathrm{V}_{\text {pin-6 }} \times\left(\frac{1-A_{\text {open-loop }}}{A_{\text {open-loop }}}\right) \approx-\mathrm{V}_{\text {pin-6 }}
$$

Overall, this topology requires only a single feedback resistor and it can achieve the targeted $500 \mathrm{kHz}$ bandwidth.

\section{B. Voltage Data Acquisition}

The voltage is measured differentially between two selected electrodes using AD8250. The potential developed gets smaller as the measuring electrode becomes further away from the current driver [7]. Dynamic gain control is employed according to the selected electrode position to enhance the system pick-up dynamic range. After band-pass filtering, the signal passes through a non-inverting gain amplifier and a THS4130 circuit, seen in Fig. 3, to offer additional gain control and anti-aliasing filtering. Then the signal is level shifted to half the ADC supply range for digitization using $\mathrm{AD} 7357$, a high performance 14-bit 4 MSPS ADC.

\section{Digital Control and I-Q Demodulation}

The digital modules include the LUT-based DDS, electrode multiplexing, ADC control, digital I-Q demodulation and UART data communication to the neural network. They implemented on a Xilinx Spartan-6 FPGA XC6SLX4. The device is operated at a $50 \mathrm{MHz}$ clock and two multiplieraccumulator (MAC) units are used for I-Q demodulation [10] with a resolution of 40-bit.

\section{Data Processing and Classifications}

After receiving the I-Q data, it is processed to obtain the impedance values which are then passed to a deep neural network for gesture classification. It consists of an auto-encoder followed by a Softmax classifier (a generalization of the binary logistic regression classifier). The auto-encoder is an unsupervised network that learns the feature representations from its input data. These learnt features are used after training by the Softmax classifier to give classification. During the training process, the data captured for training is labelled and its corresponding category is known. The neural network constantly adjusts its weights and bias in the auto-encoder and classifier through a back-propagation algorithm until it gets to the desired state. All the parameters in the neural network are stored at the end of the training process and are used for gesture recognition when a new dataset is received.

\section{RESUlts AND Discussion}

The full EIT system is shown in Fig. 4 with individual circuit blocks labelled. It was first tested on a resistive phantom, which has resistive elements changing its values from $0 \Omega$ to $128 \Omega$ with a step increase of $6.4 \Omega$. Results showed the system can detect the smallest step change.

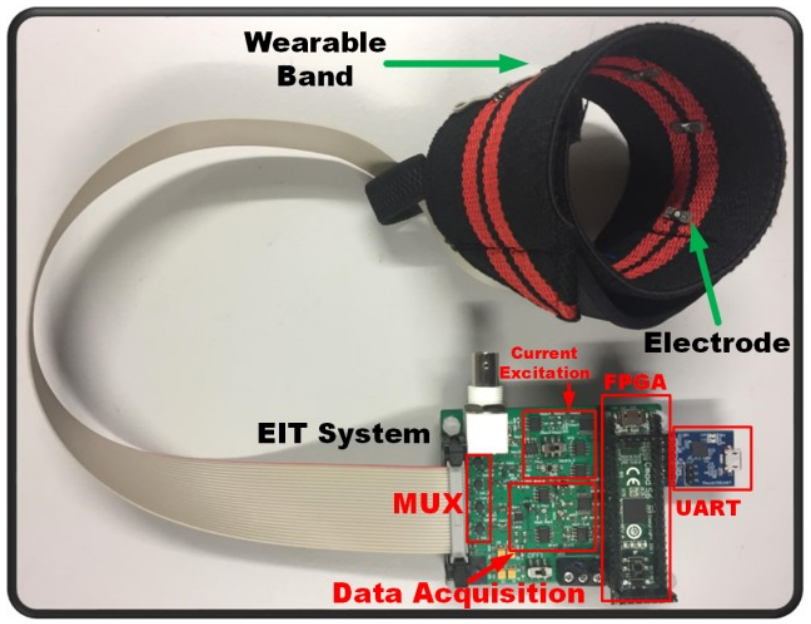

Fig. 4. Full EIT system illustration with the custom-made EIT wearable band.

Nineteen different hand gestures are designed as shown in Fig.5 with their corresponding EIT images. These coarse gestures cover hand motions from basic function, direction, and pinch motion, to number indications. The gesture recognition is divided into two phases. The first phase is the neural network training phase. After moisturizing the skin with hand cream, volunteers put on the band and perform single gesture while the system captures 50 training data sets before the user proceeds to the next gesture. Each data set contains 40 impedance values that represent the conductivity distribution of the forearm slice at the measurement instant. Classification phase begins after the neural network training is completed. The user is asked to reperform the gesture trained in the training phase. 100 data sets are taken for each gesture and accuracy is calculated as $\sigma=$ $\frac{\text { correct classfication }}{\text { total data set under test }} \times 100 \%$. The system achieves an accuracy 


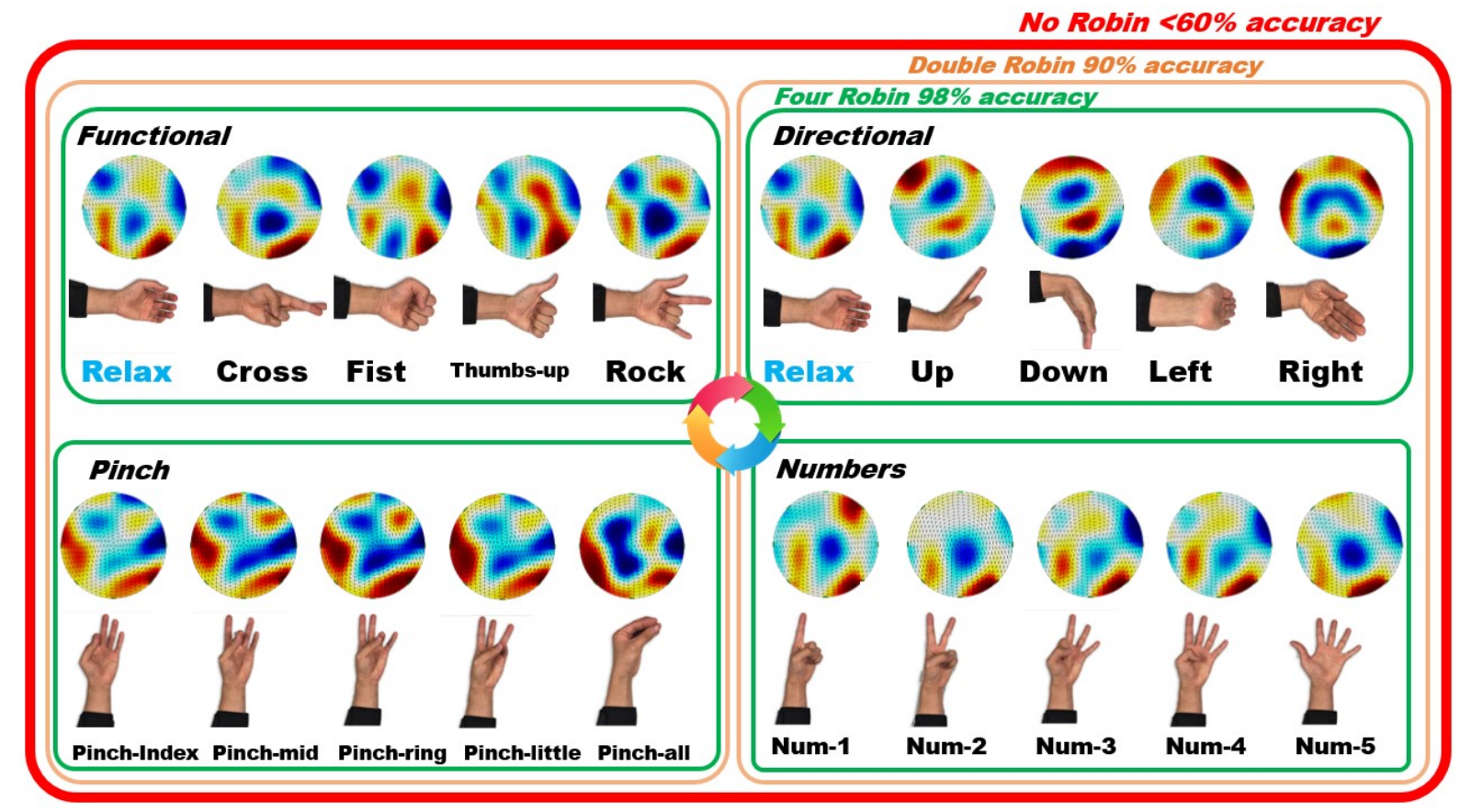

Fig. 5. Nineteen hand gestures are designed with their corresponding EIT diagrams shown above. Gestures are divided into four groups: functional gesture, directional gesture, pinch gesture and number indication gesture. With the round robin classification, the system achieves a recognition accuracy of $98 \%$.

less than $60 \%$ if all gestures are trained together. This is because certain gestures are difficult to distinguish in the same neural network, e.g. 'Cross' and 'Num-2'. Thus, it is proposed to use a round robin method to separate gestures into sub-groups as shown in Fig. 5 and to train different neural networks. This forms a round robin and the user can select sub-groups by rotating the robin e.g. using an accelerometer sensor embedded into the band (other sub-grouping methods are also reported in [4], [5]). With a double robin classification circled in orange in Fig. 5, the accuracy increased dramatically to $90 \%$ and with four-robin classification circled in green, the accuracy reaches $98 \%$.

Live recognition has also been tested. Glitch classification occurs when the user switches from one gesture to another. This is because the 'in-between gesture' is not known to the neural network. To avoid glitches, the EIT sampling speed was reduced to five data-sets/s to achieve satisfactory results.

\section{CONCLUSION}

A high performance EIT hand gesture recognition system using commercial ICs has been implemented. The system comprises an EIT hardware, a wearable electrode band, and a deep-learning neural network. It can detect a minimum of $6.4 \Omega$ impedance changes in the phantom, and can classify nineteen hand gestures with $98 \%$ accuracy using the proposed round robin method.

Future work will entail optimizing the system by exploring a more advanced round robin rotating method as well as investigating possible ways of reducing the number of robins while maintaining high classification accuracy, and possibly comparing the EIT method with other hand-gesture recognition techniques.

\section{REFERENCES}

[1] A. Fougner, Ø. Stavdahl, P. J. Kyberd, Y. G. Losier and P. A. Parker, "Control of upper limb prostheses: terminology and proportional myoelectric control - A review, "IEEE Trans. Neural Systems and Rehabilitation Engineering, 2012, pp. 663-677.

[2] K. Sridevi, M. Sundarambal, K. Muralidharan, and R. L. Josephine, "FPGA implementation of hand gesture recognition system using neural networks," 11th Int. Conf. Intelligent Systems and Control (ISCO), Coimbatore, 2017, pp. 34-39.

[3] M. Gardner, R. Vaidyanathan, E. Burdet, and B. C. Khoo, "Motion-based grasp selection: Improving traditional control strategies of myoelectric hand prosthesis," IEEE Int. Conf. Rehabilitation Robotics (ICORR), Singapore, 2015, pp. 307-312.

[4] R. Woodward, S. Shefelbine, and R. Vaidyanathan, "Integrated grip switching and grasp control for prosthetic hands using fused inertial and mechanomyography measurement," Swarm/Human Blended Intelligence Workshop (SHBI), Cleveland, 2015, pp. 1-8.

[5] Y. Zhang and C. Harrison, "Tomo: wearable, low-cost, electrical impedance tomography for hand gesture recognition," Proc. 28th Annual ACM Symp. on User Interface Software \& Technology 2015, pp. 167-173.

[6] Y. Zhang, R. Xiao, and C. Harrison "Advancing hand gesture recognition with high resolution electrical impedance tomography," Proc. 29th Annual Symposium on User Interface Software and Technology 2016, pp. 843-850.

[7] P. Langlois, Y. Wu, R. Bayford, and A. Demosthenous, "On the application of frequency selective common-mode feedback for multifrequency EIT," Physiological Measurement, vol. 36, no. 6, 2015.

[8] Y. Wu, P. Langlois, R. Bayford, and A. Demosthenous, "Design of a CMOS active electrode IC for wearable electrical impedance tomography systems," Proc. 2016 IEEE Int. Symp. Circuits and Systems (ISCAS), Montreal, QC, 2016, pp. 846-849.

[9] Y. Wu, D. Jiang, P. Langlois, R. Bayford, and A. Demosthenous, "A CMOS current driver with built-in common-mMode signal reduction capability for EIT," Proc.ESSCIRC 2017, Leuven, 2017, pp. 227-230.

[10] G. J. Saulnier et al., "An electrical impedance spectroscopy system for breast cancer detection," 2007 29th Ann. Int. Conf. IEEE Eng. in Medicine and Biology Society, Lyon, 2007, pp. 4154-4157. 